\title{
Creative Product Design of Intangible Cultural Heritage of Yi Nationality Based on QFD -TRIZ
}

\author{
Yuting Chen ${ }^{1}$, Ming Chen ${ }^{1,2}$, Jianhua Lyu ${ }^{1,2, *}$, Shilin Zhong ${ }^{1}$ and Zhenyu Wang ${ }^{1}$ \\ ${ }^{1}$ Forestry College, Sichuan Agricultural University, Chengdu 611130, China \\ ${ }^{2}$ Wood Industry and Furniture Engineering Key Laboratory of Sichuan Provincial Department of Education, Sichuan Agricultural \\ University, Chengdu 611130, China
}

\begin{abstract}
In order to inherit the intangible cultural heritage of Yi nationality and enhance the added value of the local cultural tourism products, this paper combines QFD and TRIZ to carry out research on the creative product design of intangible cultural heritage of Yi nationality. Firstly, draw technical conflicts by analyzing user needs and product technical requirements with QFD. Secondly, describe it as a standard problem using TRIZ theory, and a reasonable solution to cultural and creative product design is obtained by querying the contradiction matrix table. Finally, through design demonstration, the integration of QFD and TRIZ provides an effective and scientific method for creative product design of intangible cultural heritage of Yi nationality, and new research idea for innovative design of cultural and creative products.
\end{abstract}

\section{Introduction}

With the rapid development of social economy and the explosion of information brought by science and technology, various cultural and creative products have emerged one wave after another [1-2]. However, there are a large number of minority Intangible Cultural Heritage creative products lack of market and attention. Taking Yi Intangible Cultural Heritage creative products as an example, its development is relatively backward. With serious homogenization which renders products neither to meet the modern aesthetic standards, nor to show the characteristics of Yi customs[3]. Therefore, this paper will analyze the importance of consumer demand and the key problems of product technology with the QFD(quality model house), and then transform consumer need into product design requirement. And finally analyze the problem situation with TRIZ(Theory of the Solution of Inventive Problems), which could quickly discover the nature and contradiction of the problem, and adopt the 40 basic measures commonly used in TRIZ to solve the problem. Finally, a set of Yi Intangible Cultural Heritage creative products, which is more practical and beautiful and widely welcomed by consumers, is designed to promote local economic development and promote traditional culture.

\section{QFD and TRIZ integrated applications}

Proposed by Japanese quality management master Yoji Akao and Shigeru Mizuno, the QFD, Quality Function Deployment, aims to ensure that product design meets customer needs and values all the time. The core is to transform user needs into engineering characteristics and establish a direct mapping relationship between user needs and product design elements through quality house[4]. However, due to QFD models are limited to problem finding and analysis, it can not provide innovative ideas and tools. As a result, this paper will also utilize innovative design problem-solving tools in TRIZ to address the problem. Derived from the summary and analysis of 2.5 million patents by Genrich S.Altshuler, TRIZ is a problem-solving, analysis based on the principle of knowledge system, it is considered as a toolkit solution and provides a series of analytical methods and solutions for the problems[5-6]. The combination of QFD and TRIZ to develop products can achieve complementary advantages, accurately capture user pain points, and make the product design process clear. The application process of QFD and TRIZ integration is shown in figure 1.

*Corresponding author’s e-mail: 1jh@sicau.edu.cn 


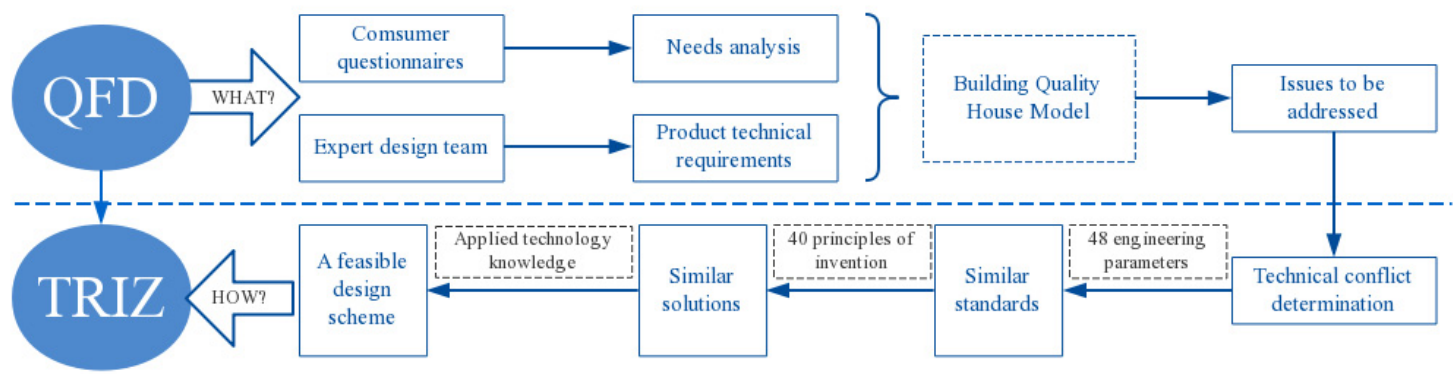

Figure 1. Flow chart of integrated application of QFD principle and TRIZ theory

\section{$3 \quad$ Building Quality Model House of Yi Intangible Cultural Heritage Creative Products}

\subsection{Demand Analysis of Yi Intangible Cultural Heritage Creative Products}

In order to make the design closer to the ideal state, 30 cultural and creative product designers, 200 tourists who came to the $\mathrm{Yi}$ area and 30 local $\mathrm{Yi}$ residents were randomly surveyed in the early stage of the design, and a total of 260 questionnaires were obtained. After summarizing the survey data, it is concluded that the needs of user experience mainly include cultural, practical, aesthetic, functional, technological, economic and publicity. In the process of product design, we grade the importance of user's demand into 7 levels and comprehensively analyze tourist demand and the design goal of tourist souvenirs. Therefore, we can design and produce according to the importance of each customer's demand[7]. According to the design method and quality requirement of cultural and creative product, the technical requirements of Yi's cultural and creative products are summarized based on the principle of macro and pertinence, and 10 technical requirements are obtained: modeling rich in Yi's Intangible Cultural Heritage cultural characteristics, reasonable color match, high compressive strength, suitable material, functional diversification, small size, beautiful shape, easy processing, controllable cost and stable structure.

\subsection{Building a Quality Model House}

According to the research data, the quality house of $\mathrm{Yi}$ Intangible Cultural Heritage creative products is constructed within the framework of QFD theory, and the importance of user demand and technical demand is scored into the quality house to obtain a comprehensive score. It can be concluded that the technical requirements of Yi Intangible Cultural Heritage cultural and creative souvenirs are ranked in order of importance, and the three technical indexes in the top three are taken as the key product technical requirements, which are: "modeling rich in Yi Intangible Cultural Heritage cultural characteristics", "functional diversification"and "small size", as shown in figure 2. 


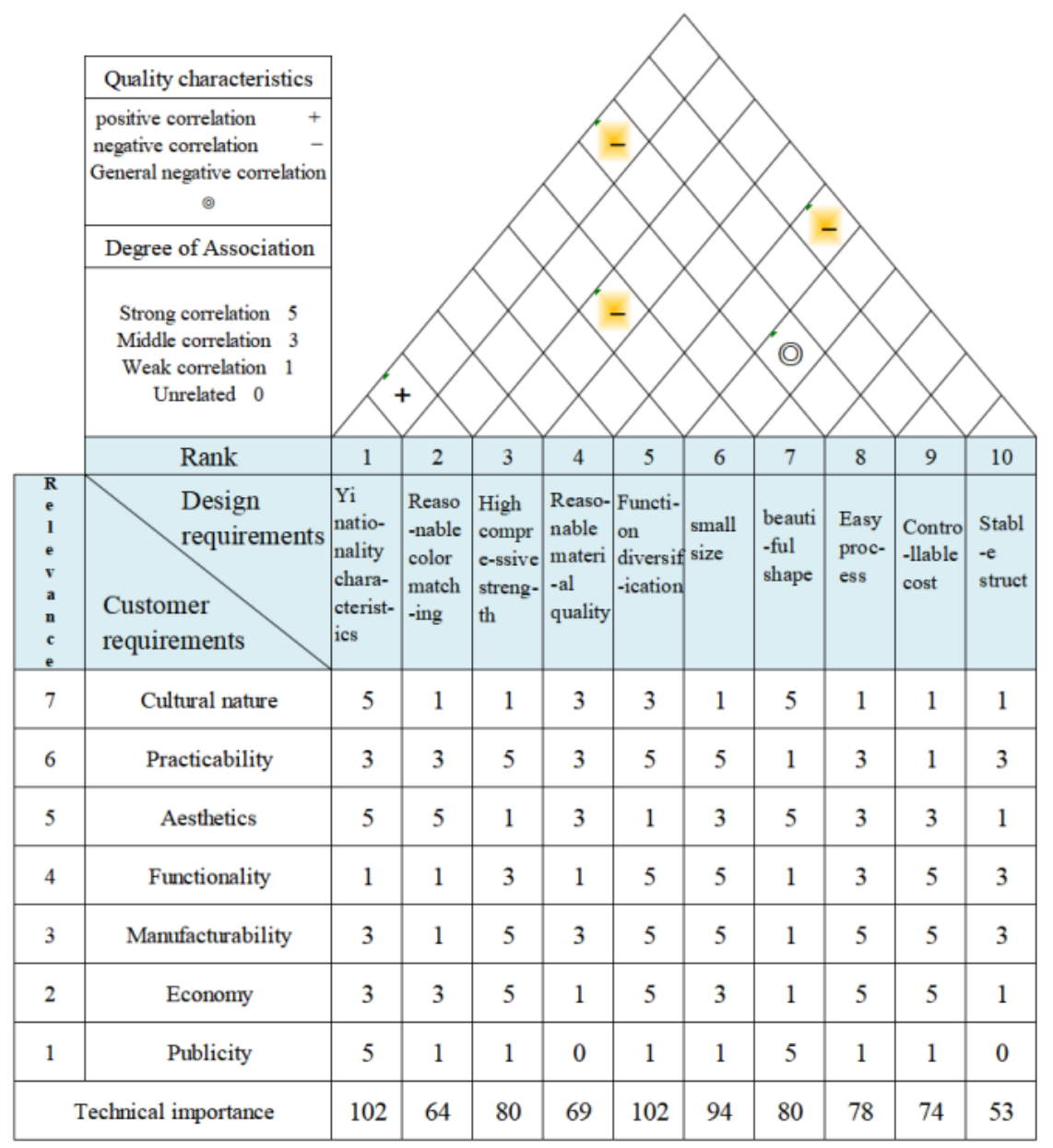

Figure 2. Quality Model House for Intangible Cultural Heritage Products

\subsection{Technical Contradictions of Yi Intangible Cultural Heritage Creative Products}

This paper analyzes the roof matrix of $\mathrm{Yi}$ cultural and creative product quality house, seeks the interrelation between the technical requirements, and determines the technical conflict[8]. Three important technical conflicts can be concluded: there is a strong negative correlation between "modeling rich Yi Intangible Cultural Heritage cultural characteristics"and "easy processing"; there is a strong negative correlation between "functional diversification"and "stable structure"; there is a negative correlation between "small size"and "high compressive strength".

\section{$4 \quad$ Analysis of Design Contradiction under TRIZ}

Regarding the contradiction between the above three pairs of actual processing technology transform it into the conventional TRIZ standard technical contradiction, and then refer to the TRIZ contradiction matrix table of version 03[9]. The 48 improved general engineering parameters are corresponding to 40 invention principles, and the design scheme model for solving the problem is obtained in the matrix.(Table 1)

To solve the contradiction between "modeling rich in Yi's Intangible Cultural Heritage cultural characteristics"and "easy processing", we should improve "aesthetic"parameters, but "manufacturability"will deteriorate. The following design examples will be designed using the above principles: NO.1(segmentation), NO.2(extraction), NO.6 (generality), NO.10(pre-operation), NO.13(reverse), NO.16(not reach or exceed function), NO.22(change harm to benefit), NO.25(self-service). The other two pairs of contradictions use the same step.

Table 1 Three pairs of Technical Contradictions and Corresponding Solutions

\begin{tabular}{cccl}
\hline & Technical conflicts & Contradictions in TRIZ & Solutions \\
\hline & Yi nationality characteristics & Beautiful & $\begin{array}{l}\text { NO.1( segmentation) } \\
\text { NO.2( extraction) } \\
\text { NO.6( versatility) ... }\end{array}$ \\
\hline & Easy to process & Manufacturability & NO.4(asymmetry) \\
\cline { 3 - 4 } & Small size & Volume of moving objects &
\end{tabular}




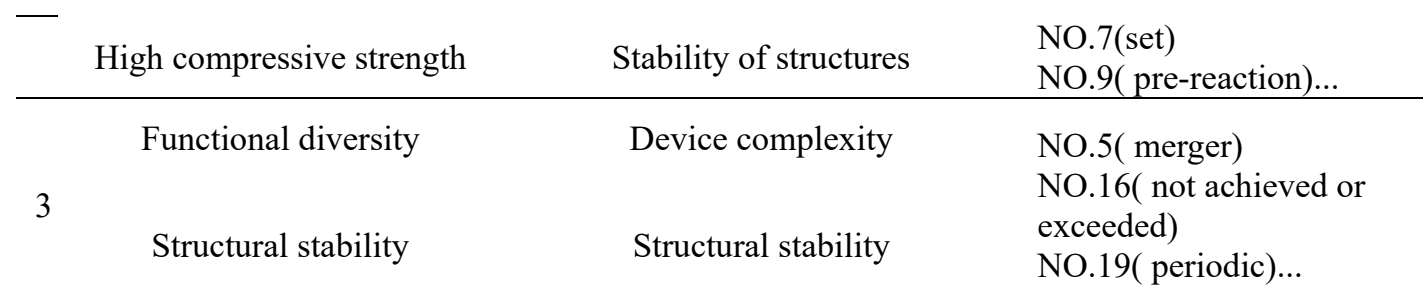

\section{Demonstration of product design of intangible cultural heritage of Yi nationality}

\subsection{Re-engineering of cultural elements of Intangible Cultural Heritage of Yi Nationality}

Nowadays, the development of Yi tourism industry chain is not enough, and the development of $\mathrm{Yi}$ Intangible Cultural Heritage cultural creation products lacks the local cultural characteristics of Yi nationality group. On the one hand, Yi Intangible Cultural Heritage production enterprises generally have insufficient technical funds, small scale and not high management level, which result their inability of launching cultural and creative products with strong local cultural characteristics[3]. On the other hand, the single variety of Yi cultural and creative which lacks the collection and appreciation value of cultural and creative souvenirs on the market, limits the tourists' consumption desire and further restricts the benign development of the local industrial chain. Only the characteristic $\mathrm{Yi}$ cultural and creative products will attract the attention and stimulus users' purchase desire.
On the basis of the contradiction matrix, it is concluded that the "Yi characteristics"and "processing technology"correspond respectively to the "beauty"and "manufacturability" of 48 general engineering parameters. Provided by the matrix, the invention principle is numbered NO.1( segmentation), NO.2( extraction), NO.6( general), NO.10( pre-operation), NO.16( failure to achieve or exceed the function) and NO.22( change damage to profit). Aiming at this pair of contradictions, simplify and refine the design, decoration, ribbon and other elements of Yi triangle bag through the principle of invention NO.2 extraction. And then combine the unique elements of $\mathrm{Yi}$ nationality group with cultural and creative products to ensure the practicability and decoration of cultural and creative products. By using the principle NO.6 generality of invention, the product is divided into components which are easy to assemble and disassemble, so that a product has many functions. And replace the traditional handmade high cost materials with ordinary materials that can be processed in large quantities according to the principle of material replacement. Such design not only the iconic features of the Yi triangle package, but also achieves the effect of simplifying the process steps and combine the traditional culture of Yi nationality with modern life (Figure 3).
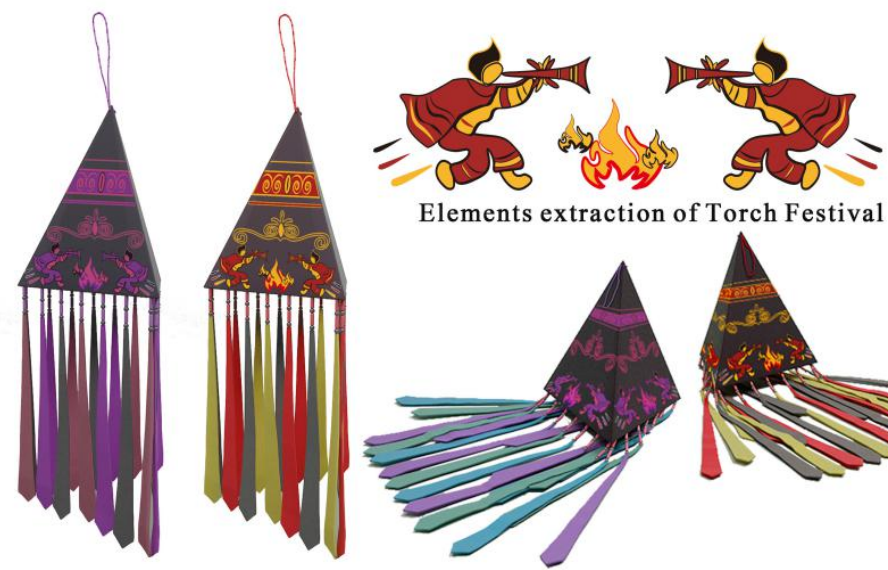

Elements extraction of Torch Festival
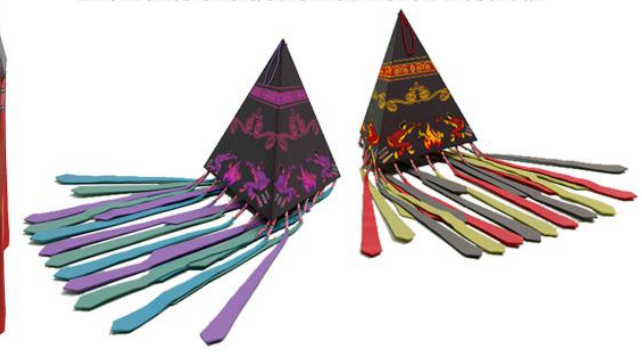

Figure 3. Redesign of the Yi nationality's elements in the triangle bag

\subsection{People-centred design philosophy}

With the goals of creativity and life practicality, most of the cultural and creative products are in the design of relatively small, light, beautifully packaged, creative[10]. The development of such creative products is the best choice for foreign tourists to bring home or give as gifts to friends, and is also one of the important carriers for the extension of Yi Intangible Cultural Heritage. When the product meets the characteristics of ingenuity and lightness, it is necessary to reduce its volume. However, meanwhile the strength of the product against external forces will be reduced, causing the increasing probability of deformation and damage.

Aiming at the contradiction between "volume of moving object"and "strength", first of all, considering from the angle of inclusion, rotation, vertical and horizontal through the principle of NO.7 suit, nest and design the components of cultural and creative product, so that one component can penetrate into another original. Apply the principle of NO.9 pre-addition reaction principle and NO.40 composite material, and utilize nylon cloth, PVC, PU and other materials with strong elasticity, comfortable hand feeling, excellent compression resistance, mechanical properties, and 
relatively low production cost, wide application range, and easy processing. Finally, by applying the NO.15 number dynamic principle, the inclusion package is divided into different parts, some of which can be expanded, combined, or folded up. Folding up will also reduce the volume, make it moveable, and make the

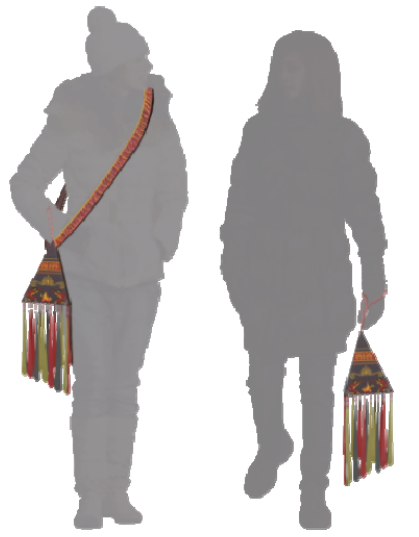

Figure 4. How to use the Triangular Packet

\subsection{Function optimization design}

Through the analysis of the questionnaire, the majority of the tourist are middle-aged people who have high expectations on the function of the cultural and creative products. If the diversification of product functions is increased, which means that the complexity of cultural and creative product devices will be enhanced, the stability of souvenir structure will be reduced.

Aiming at the contradiction between "device complexity"and "structural stability", the invention principle NO.24 the intermediary principle are used to divide the product into components which are easy to assemble and expand, so that a product has many functions. Considering the placement problem after use, the shape of $\mathrm{Yi}$ triangle bag is changed from two-dimensional to three-dimensional by using the principle of NO.36 phase state conversion and NO.2 extraction, expanding the capacity of the product, and reconstructing the internal hollowing, increasing the storage function and improving its practicability. We need to combine the principle of NO.2, NO.24 and NO.36 invention skillfully. The shape of the triangle package relative to the plane is first converted into three-dimensional, and then the interior is accurately divided into the area of placing items, such as lipstick area and mobile phone area; at the same time, the stability of product placement which not in use is guaranteed. That is to meet the needs of users, and achieve the functional value of the package. The functional diversity is shown in Figure 5.

\section{Conclusion}

This paper applies the QFD and TRIZ integrated methods to carry out the research of Yi Intangible Cultural Heritage creation product design. The innovation point of Yi Intangible Cultural Heritage creation product is found accurately by using the QFD to explore the contradiction shape more stretch and beautiful. The handbag designed after this operation is exquisite and chic, emphasizing "light, thin, short and small", which is convenient for tourists to travel and carry. User usage is shown in Figure 4.

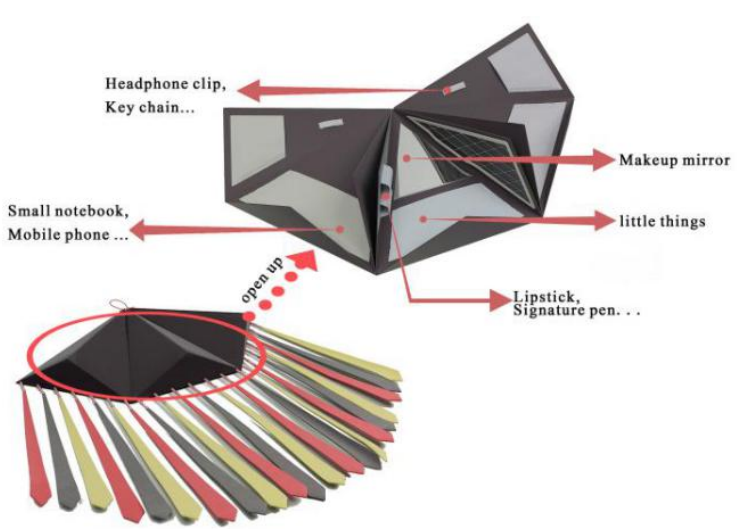

Figure 5. Interior Expansion Diagram

conflict between the user and the product technology in the process of interaction and querying the contradiction matrix table to resolve the conflict in the system. Through the demonstration of triangle package redesign, this method can effectively solve the innovative design problems of Yi Intangible Cultural Heritage cultural and creative products in practical, aesthetic and functional aspects. It can also better meet the needs of users, provide a scientific method for $\mathrm{Yi}$ Intangible Cultural Heritage cultural and creative product design, and hopefully provide new research ideas for the design of other similar products in the market. Continue innovation and optimization, and solve the product process, details, follow-up design and other problems from the user needs. Finally paying attention to the user experience is the main work of the next step.

\section{Acknowledgments}

The authors are grateful for the support of the Ministry of Education Humanities and Social Sciences Research Project of China (Grant No. 19YJC760009), Key Research and Development Project of Sichuan Science and Technology Plan Projects (Grant No. 2020YFS0357), Project of Modern Design and Culture Research Center, Sichuan Key Research Base of Philosophy and Social Sciences (Grant No. MD18Z002) and Opening Foundation for Industrial Design Industry Research Center, Key Research Base of Humanities and Social Sciences, Sichuan Education Department (Grant No. GYSJ18-037).

\section{References}

1. Cao Zhenzhen. Creative Product Development and Design Based on Intangible Cultural Heritage [J]. Industry and Technology Forum, 2019, 18(22): 53-54.

2. Xiang Yong. A Pioneer Experiment of Inheriting Excellent Traditional Culture [J]. People's Forum, 
2019(09): 124-126.

3. Zhao Qin, Li Yumin. Extraction and Application of Yi Decorative Patterns in Tourist Souvenirs [J]. Packaging Engineering, 2017, 38(20): 240-244.

4. Fanglan Zhang, Minglang Yang, Weidong Liu. The Evaluation Method of Characteristics Priority of Automobile Modeling Design Based on QFD [J]. Packaging Engineering, 2014, 35(24): 59-62.

5. Shao Yunfei, Wang Simeng, Zhan Kun. Review on TRIZ Theory Integration and Application [J]. Journal of the University of Electronic Science and Technology (Social Sciences Edition), 2019, 21(04): 30-39.

6. GADD K. TRIZ Problem-Solving Maps and Algorithms[J]. TRIZ for Engineers : Enabling Inventive Problem Solving, 2011: 419-450.

7. Yang Jing. The Creative Design of Shaanxi Tang Culture Tourism Souvenir Based on QFD and TRIZ [J]. Packaging Engineering, 2017, 38(14): 203-207.

8. Wang Junhua, Peng Huatao. Effect of Collaborative Innovation Consciousness on Staff's Innovation Ability in TRIZ Application [J]. Science and Technology Progress and Countermeasures, 2015, 32(09): 146-151.

9. Han Ruiyun. Research on Product Innovation Design Based on QFD and TRIZ Integration [D]. Hebei University of Technology, 2015.

10. Zhang Yao. A Study on the Development and Design of Cultural and Creative Products Based on Museum Resources [D]. Suzhou University, 2015. 